were employed at a single DOE nuclear site that implemented the DOE beryllium rule between 1999-2002. Workers were classified as BeS when they met one of the following definitions: two abnormal blood BeLPT results, one abnormal and one borderline blood BeLPT result, three borderline blood BeLPT results, or one abnormal lung lavage BeLPT result. Descriptive statistics, longitudinal analyses, and correlation analyses were utilized to evaluate the trends in incidence before and after implementation of the beryllium rule.

Results Results indicated a general decrease in exposure and $\mathrm{BeS}$ incidence rates, though there are some years with notable increases presumably due to increased clean-up activity, where construction and decommissioning workers had opportunity for exposure from legacy beryllium operation materials that were not part of normal current production activity.

Conclusion These findings provide support for the hypothesis that the DOE Beryllium Rule helps prevent beryllium sensitization. Future research will more precisely assess the relationship between beryllium exposure levels and beryllium incidence over time.

\section{O4D.3 BONE LEAD ASSOCIATIONS WITH BLOOD LEAD, KIDNEY FUNCTION, AND BLOOD PRESSURE AMONG U.S., LEAD- EXPOSED WORKERS IN A SURVEILLANCE PROGRAM}

${ }^{1}$ Vaughn Barry, ${ }^{2}$ Andrew Todd, ${ }^{1}$ Kyle Steenland*. ${ }^{1}$ Rollins School of Public Health, Emory U, Atlanta, USA; ${ }^{2}$ Mt Sinai School of Medicine, NY, US

\subsection{6/OEM-2019-EPI.103}

Objectives Bone lead and past blood lead (BL) levels may be more strongly associated with current health effects than current $\mathrm{BL}$, representing recent exposure. We examined whether current bone lead is correlated with maximum past BL, and compared three measures of lead as predictors for current blood pressure (BP) and kidney function among workers with past occupational exposure to lead.

Methods Adult men in a lead surveillance program living within 200 miles of New York City were enrolled in this observational study during 2016-2017. We gathered data on current bone and BL, BP, and estimated glomerular filtration rate (eGFR). Maximum past BL was obtained from prior surveillance program data. Regression models were used to determine associations of health endpoints with different measures of lead.

Results Among 211 participants, median (interquartile range) bone lead, maximum past BL, and current BL were $13.8(9.4-19.5) \mu \mathrm{g}$ of lead per gram of bone mineral, 29.0 $(14.0-38.0) \mu \mathrm{g} / \mathrm{dl}$, and $2.5(1.5-4.4) \mu \mathrm{g} / \mathrm{dl}$, respectively. Both maximum past and current BL were significantly associated with current bone lead in adjusted analyses $(\mathrm{p}<0.0001$ for both), with associations driven by those with the highest BL levels. Bone lead was associated with increased systolic BP $(p=0.02$, model R-square $=0.16)$, but quartile analyses were not monotonic. Bone lead was also non-significantly associated with decreased (worse) eGFR (regression coefficient $=-0.15, \quad \mathrm{p}=0.18$, model $\mathrm{R}$ square $=0.28$ ).

Conclusions Bone lead was significantly associated with past maximum and current BL. Bone lead, but not past maximum or current BL, was associated with elevated systolic BP.
O4D.4 CHANGES IN KIDNEY FUNCTION AMONG SUGARCANE CUTTERS ON A MODERATELY HOT SUGAR PLANTATION IN SOUTH AFRICA

1,2 Mollen Magombo*, ${ }^{3}$ Lars Barregard, 1,2Spo Kgalamono, 2,4 Jaya George, ${ }^{2}$ Saraladevi Naicker, ${ }^{5}$ Elton Dorkin, ${ }^{1,2}$ Felix Made, ${ }^{1,2}$ David Rees. ${ }^{1}$ National Institute For Occupational Health, NHLS, Johannesburg, South Africa; ${ }^{2}$ University of the Witwatersrand, Johannesburg, South Africa; ${ }^{3}$ National Health Laboratory Service, Johannesburg, South Africa; ${ }^{4}$ Occupational and Environmental Medicine, University of Gothenburg, Gothenburg, Sweden; ${ }^{5}$ Dr EP Dorkin and Associates (Pty) Ltd, Durban, South Africa

\subsection{6/OEM-2019-EPI.104}

Introduction Epidemic chronic kidney disease of unknown cause $(\mathrm{CKDu})$ occurs among sugar cane workers, notably cane cutters doing heavy manual work in hot tropical environments in Mesoamerica. Repetitive dehydration consequent on strenuous work in heat is posited as the cause. A Nicaraguan cohort study showed remarkable kidney function decreases across six weeks of the cane cutting season consistent with the dehydration hypothesis. This Nicaraguan study was replicated on a sugar plantation in South Africa about $3500 \mathrm{kms}$ from the equator and cooler than previous study locations to examine whether less extreme ambient conditions resulted in reduced kidney stress.

Methods 38 cane cutters and 36 referents of similar socio-economic status but doing less strenuous work on the same plantations provided pre- and post-shift blood and urine samples for measures of kidney function and hydration on Day 1 and Week 9 of the cutting season. Frozen specimens were transported to a chemical pathology laboratory in Johannesburg for analysis. Parameters measured included cystatin C, eGFR (derived from cystatin C), serum creatinine, serum neutrophil gelatinase-associated lipocalin (NGAL), serum uric acid and osmolality.

Results Minimum and maximum temperatures were 20.9 and 26.5o C on Day 1 and 13.6 and 26.6 o C on Week 9. There were clinically modest, albeit statistically significant, increases in mean cystatin $\mathrm{C}$ pre-shift values between Day 1 and Week 9 in the cane cutters: 0.847 to $1.011 \mathrm{mg} / \mathrm{L}(\mathrm{p}=0.00)$ which means a considerable decline in eGFR. Other results were not consistent among the various markers of effect on the kidneys. The magnitude of the increase in cystatin $\mathrm{C}$ among referents was similar to the cutters: 0.771 to $0.904 \mathrm{mg} / \mathrm{L}$.

Conclusions Kidney function markers seemed to be much less affected in the cooler study location than in the hotter one.

\section{O4D.5 LEFT SIDED HEARING LOSS AMONG HEAVY EQUIPMENT OPERATORS (HEOS) IN MINING INDUSTRY}

Vishnumohan Janardhanam*, Akila Vishnumohan*, MG Karthic. JSW Steel Limited, Salem, India

\subsection{6/OEM-2019-EPI.105}

Introduction Open surface mining is utilized to extract limestone for the production of cement. Using heavy motor vehicles (HMVs) and other earthmoving equipment, skilled personnel [HEOs] perform this operation. HEOs are exposed to significant noise (80-90 dBA) emitted by these HMVs for about 8 hours a day. We report the results of pure tone audiometry (PTA) conducted on HEOs as part of a health surveillance programme.

Methods In this observational study, 108 HEOs working in a large scale mining industry in South India were assessed for 